\title{
Plant Sources
}

National Cancer Institute

\section{Source}

National Cancer Institute. Plant Sources. NCI Thesaurus. Code C18944.

Indicates that a product is derived from a plant. 